\title{
The impact of medication-related osteonecrosis of the jaws on the quality of life in cancer patients
}

\author{
Raquel-D'Aquino-Garcia Caminha ${ }^{1}$, Patricia-Lopes Alcantara ${ }^{1}$, Caroline-Gomes Carvalho ${ }^{1}$, Verônica-Caro- \\ line-Brito Reia ${ }^{2}$, Ana-Lucia-Alvares Capelozza ${ }^{3}$, Paulo-Sérgio-da Silva Santos ${ }^{4}$
}

\author{
${ }^{1}$ MSc, DDS, Department of Surgery, Stomatology, Pathology and Radiology, Bauru School of Dentistry, University of São Paulo, \\ Bauru, Brazil \\ ${ }^{2}$ DDS, Center Research Clinical, University of São Paulo, Bauru, Brazil \\ ${ }^{3} \mathrm{PhD}, \mathrm{MSc}$, DDS, Department of Surgery, Stomatology, Pathology and Radiology, Bauru School of Dentistry, University of São \\ Paulo, Bauru, Brazil \\ ${ }^{4} \mathrm{PhD}$, MSc, DDS, Department of Surgery, Stomatology, Pathology and Radiology, Bauru School of Dentistry, University of São \\ Paulo, Bauru, Brazil
}

Correspondence:

Alameda Octávio Pinheiro Brisola

9-75, Postal Code: 17012-901

Bauru, São Paulo, Brazil

paulosss@fob.usp.br

Caminha RDG, Alcantara PL, Carvalho CG, Reia VCB, Capelozza ALA, Santos PSS. The impact of medication-related osteonecrosis of the jaws on the quality of life in cancer patients. J Clin Exp Dent. 2020;12(8):e725-9.

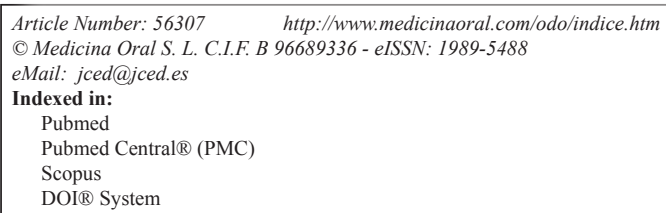

\begin{abstract}
Background: To evaluate the impact of oral health on the quality of life (QOL) of individuals undergoing cancer treatment at the time of diagnosis of medication-related osteonecrosis of the jaw (MRONJ).

Material and Methods: The present cross-sectional study analyzed patients with MRONJ from 2013 to 2019. The collected data included demographic data, base disease, medications associated with MRONJ, route of administration and time of use, signs, symptoms, and tomographic features of acute MRONJ, staging according to American Association of Oral and maxillofacial Surgeons position paper 2014 (AAOMS), type of dental treatment performed, outcome, and the responses to the Oral Health Impact Profile questionnaire (OHIP-14). Statistical analysis was performed using the Tukey test to study the association between oral condition and the QOL. A p-value of less than 0.05 was considered statistically significant.

Results: The sample consisted of 16 medical records of patients with MRONJ. Psychological discomfort showed alarmingly significant results $(p<0.001)$ with strong negative impact on the QOL of the patients. Functional limitation was the least affected dimension $(p=0.747)$. The other dimensions did not show statistically significant results. Conclusions: MRONJ compromises oral health and negatively impacts the QOL, especially with respect to the psychological discomfort (worry and stress). The OHIP-14 questionnaire proved to be an effective tool in the assessment of this impact.
\end{abstract}

Key words: Medication-related osteonecrosis of the jaw, quality of life, oral health, OHIP-14. 


\section{Introduction}

Medication-related osteonecrosis of the jaw (MRONJ) is associated with symptoms, such as halitosis, severe pain, difficulty in chewing, and dysphagia, that have a negative impact on the quality of life (QOL) $(1,2)$. The QOL indicators are related to physical, social, and psychological well-being and can be assessed through generic or specific questionnaires according to the type of disease (3).

The Oral Health Impact Profile questionnaire (OHIP-14) (4) has been widely used $(5,6)$ and allows the self-assessment of the individual with respect to the impact of oral health on the QOL. The OHIP-14 is a questionnaire recognized for its sensitive potential for interference in the QOL. It is a low-cost, easily applied tool, and it allows a greater access to the population. Consequently, it helps in identifying the effects on the QOL of individuals, enabling the best targeted treatment. Though studies have reported a high prevalence of MRONJ $(7,8)$, very few have reported its impact on the $\operatorname{QOL}(5,9,10)$. According to the SF-12 questionnaire that evaluates the general QOL (2), MRONJ is a condition that can lead to worsening of the physical and mental state of patients. Treatment for MRONJ aims to restore the QOL by resolving the symptoms in the jaws (11).

Researchers have pointed out the scarcity in the literature about oral health impairment and the QOL of cancer patients (10,13-16). A high incidence of suicides has been demonstrated in cancer patients, probably due to the functional alterations that drastically affect the QOL (17). Therefore, the importance of preserving the QOL of these individuals through prevention and control of MRONJ $(2,5,16)$ becomes crucial.

The aim of this study was to evaluate the impact of oral health on the QOL in individuals undergoing cancer treatment at the time of diagnosis of MRONJ.

\section{Material and Methods}

The present cross-sectional study was conducted at a clinical research center specializing in care for systemically compromised individuals through the analysis of 902 medical records of patients who underwent dental treatment from 2013 to 2019. Sixteen medical records of patients with MRONJ were selected. This study was approved by the Research Ethics Committee of the University of São Paulo (CAAE: 65053617.0.0000.5417).

Demographic data collected from the 16 medical records included name, gender, age, base disease, medications associated with MRONJ, route of administration and time of use, signs, symptoms, and tomographic features of acute MRONJ, staging according to the norms by AAOMS (1), type of dental treatment performed, outcome of MRONJ, and impact of oral health on the QOL.

The portuguese version (18) of the OHIP-14 was used as the QOL questionnaire in which 7 dimensions were divided into 14 questions with responses attributed by the individuals through a Likert scale (0 to 4 ) to quantify the impact of oral health on the QOL. The value of the answers was multiplied by the power corresponding to each question to calculate the total value for each dimension.

The seven dimensions included functional limitation, physical pain, psychological discomfort, physical disability, psychological disability, social disability, and disability. Values for each dimension were calculated and the impact of each dimension was classified as low $(0-1.33)$, medium $(1.33-2.68)$, or high $(>2.68)$. According to the total values the correlation was classified as having weak impact $(<9.33)$, medium impact $(9.33$ $18.66)$, or strong impact $(>18.66)$. Higher values suggested greater negative impact of oral health on the QOL of the patients. Statistical analysis of the obtained data was performed using the Tukey test to study the correlation between oral condition and the QOL. Results were considered statistically significant when the $\mathrm{p}$-value was less than 0.05 .

\section{Results}

Tables 1, 2, and 3 show the demographic data and the base diseases or metastases, the clinical / imaging characteristics of patients with MRONJ, and the characteristics related to osteonecrosis-associated drugs.

The dimension related to psychological discomfort (D3), which evaluated the degree of concern and stress, showed the most significant $(p<0.001)$ strong negative impact on the QOL of the individuals under study. The dimension related to functional limitation (D1), which assessed the impact on word pronunciation and dysgeusia, showed the least impact ( $p=0.747)$ on the QOL. No significant results were observed for the other dimensions $(p>0.05)$. The values obtained from the OHIP-14 according to the dimensions are listed in table 4 .

\section{Discussion}

Demographic factors such as age, gender, and location of the neoplasm may interfere with the development of MRONJ. In the present study, 9 (56.25\%) cases were female. The relatively higher incidence of the disease in females may be due to a higher frequency of diseases such as breast cancer and osteoporosis in women (1), and subsequently, greater indication of the drugs that may be responsible for MRONJ. Mandible was the most affected region (10 [62.5\%] cases) followed by the maxilla (6 [37.5\%] cases). These findings can be explained by the local vascular characteristics. Since the mandible has less vascularization when compared with the maxilla (16), the incidence of osteonecrosis may be higher. The OHIP-14(18) questionnaires were applied at the time of the diagnosis of MRONJ. The responses showed alarming negative results for the dimension of psycho- 
Table 1: Demographic data (age, sex, type / location of cancer, and occurrence of metastasis)

\begin{tabular}{|l|c|}
\hline \multirow{4}{*}{ Gender } & Female: $9(56,25 \%)$ \\
\cline { 2 - 2 } & Male: $7(43,75 \%)$ \\
\hline \multirow{4}{*}{ Age (years) } & Minimum: 39 \\
\cline { 2 - 2 } & Average: 64,7 \\
\cline { 2 - 2 } & Median: 64,5 \\
\hline \multirow{5}{*}{ Base Disease (location) } & Maximum: 80 \\
\cline { 2 - 2 } & Breast: $8(50 \%)$ \\
\cline { 2 - 2 } & Renal: $3(18,75 \%)$ \\
\cline { 2 - 2 } & Prostate: $3(18,75 \%)$ \\
\cline { 2 - 2 } & Multiple Myeloma: $1(6,25 \%)$ \\
\hline Metastases (location) & Straight: $1(6,25 \%)$ \\
\cline { 2 - 2 } & Total: $10(62,5 \%)$ \\
\cline { 2 - 2 } & Bone: $6(37,5 \%)$ \\
\cline { 2 - 2 } & Pulmonary: $2(12,5 \%)$ \\
\cline { 2 - 2 } & Hepatic: $1(6,25 \%)$ \\
\cline { 2 - 2 } & Bowel: $1(6,25 \%)$ \\
\hline
\end{tabular}

Table 2: Clinical and imaging characteristics of patients with MRONJ.

\begin{tabular}{|c|c|}
\hline \multirow[t]{2}{*}{ Location } & Maxila: $6(37,5 \%)$ \\
\hline & Mandible: $10(62,5 \%)$ \\
\hline \multirow[t]{6}{*}{ Signs/ Symptoms } & Exposed bone: $15(50 \%)$ \\
\hline & Pain: $6(20 \%)$ \\
\hline & Asymptomatic: $6(20 \%)$ \\
\hline & Suppuration: $2(6,67 \%)$ \\
\hline & No clinical sign: $1(3,33 \%)$ \\
\hline & Not reported: 3 cases \\
\hline \multirow[t]{6}{*}{ Imaging characteristics (MRONJ sites) } & Nonhealing extraction sockets: $9(60 \%)$ \\
\hline & Irregular borders: $2(13,33 \%)$ \\
\hline & Suggested bone sequestration: $2(13,33 \%)$ \\
\hline & Bone esclerosis: $1(6,67 \%)$ \\
\hline & No change: $1(6,67 \%)$ \\
\hline & Not reported: 4 cases \\
\hline \multirow{9}{*}{$\begin{array}{l}\text { Imaging characteristics (sites without clinical } \\
\text { signs of MRONJ) }\end{array}$} & Bon esclerosis: $6(31,58 \%)$ \\
\hline & Nonhealing exraction sockets: $4(21,05 \%)$ \\
\hline & Increased periodontal ligament space: $3(15,80 \%)$ \\
\hline & Lamina dura thickening: $2(10,53 \%)$ \\
\hline & Buco-sinus communication: $1(5,26 \%)$ \\
\hline & Cortical irregularities: $1(5,26 \%)$ \\
\hline & Bone trabecular hypodensity: $1(5,26 \%)$ \\
\hline & Hyperdensity around implant: $1(5,26 \%)$ \\
\hline & Nor reported: 4 cases \\
\hline \multirow[t]{4}{*}{ Staging (AAOMS 2014) } & $0: 1(6,25 \%)$ \\
\hline & I: $9(56,25 \%)$ \\
\hline & II: $6(37,5 \%)$ \\
\hline & III: $0(0 \%)$ \\
\hline
\end{tabular}

'MRONJ: Medication-related osteonecrosis of the jaw

*AAOMS: American Association of Oral and Maxillofacial Surgeons 
Table 3: Types of MRONJ广-associated drugs.

\begin{tabular}{|l|c|}
\hline \multirow{4}{*}{ Medications } & Zoledronic Acid: $10(62,5 \%)$ \\
\cline { 2 - 2 } & Pamidronate: $3(18,75 \%)$ \\
\cline { 2 - 2 } & Sunitinib: $1(6,25 \%)$ \\
\cline { 2 - 2 } & Pazopanib: $1(6,25 \%)$ \\
\cline { 2 - 2 } Route of administration & Zoledronic acid + Pamidronate: $1(6,25 \%)$ \\
\cline { 2 - 2 } & Intravenous: $14(87,5 \%)$ \\
\hline Usage time (months) & Oral: $2(12,5)$ \\
\cline { 2 - 2 } & Minimum: 6 \\
\cline { 2 - 2 } & Medium: 36 \\
\cline { 2 - 2 } & Maximum: 108 \\
\hline
\end{tabular}

${ }^{\dagger}$ MRONJ: Medication-related osteonecrosis of the jaw

Table 4: Results after application of the OHIP-14† questionnaire according to the total value and the 7 dimensions.

\begin{tabular}{|c|c|c|c|c|c|c|c|}
\hline Total & D1/LF & D2/PP & D3/PD* & D4/PD & D5/PD & D6/SD & D7/D \\
\hline 19.86 & 1.47 & 4 & 4 & 1.56 & 3 & 2.24 & 3.59 \\
\hline 9.91 & 1.96 & 1.66 & 3.55 & 1.56 & 0 & 0 & 1.18 \\
\hline 1.24 & 0 & 0.34 & 0.9 & 0 & 0 & 0 & 0 \\
\hline 6.05 & 0.98 & 1.66 & 1.1 & 0.96 & 0 & 0.76 & 0.59 \\
\hline 7.7 & 0 & 0.68 & 0.9 & 1.48 & 1.6 & 1.86 & 1.18 \\
\hline 15.67 & 0.98 & 2.68 & 3.1 & 2.52 & 1.8 & 2 & 2.59 \\
\hline 14.1 & 0.51 & 2.98 & 4 & 1.56 & 2.2 & 0.62 & 2.23 \\
\hline 14.48 & 2.98 & 1.98 & 4 & 1.04 & 0 & 2.48 & 2 \\
\hline 17.41 & 1.47 & 2.66 & 4 & 2.08 & 1.6 & 3.24 & 2.36 \\
\hline 9.32 & 0.98 & 1.32 & 4 & 1.04 & 0.8 & 0 & 1.18 \\
\hline 3.83 & 0 & 0.34 & 1.45 & 0 & 0.8 & 1.24 & 0 \\
\hline 11.22 & 0.49 & 1.32 & 2.9 & 0.52 & 1.6 & 2.62 & 1.77 \\
\hline 22.35 & 1.96 & 4 & 4 & 3.52 & 2.8 & 2.48 & 3.59 \\
\hline 9.92 & 0.98 & 1.98 & 1.8 & 0.48 & 2.2 & 2.48 & 0 \\
\hline 0.68 & 0 & 0.68 & 0 & 0 & 0 & 0 & 0 \\
\hline 18.17 & 0 & 3.34 & 3.1 & 2.52 & 2.8 & 3 & 3.41 \\
\hline
\end{tabular}

"Statistically significant $(P<0.05)$

${ }^{\dagger}$ The Oral Health Impact Profile questionnaire

D1 to D7: Dimensions, LF: Functional Limitation, PP: Physical pain, D3/PD: psychological discomfort, D4/PD: physical disability,

D5/PD: psychological disability, SD: social disability, D: Disability

logical discomfort in which the highest score regarding oral health concerns and stress was observed. After comparison among the dimensions of functional limitation (D1), physical pain (D2), and psychological discomfort (D3), highest scores were seen for D3 followed by D2. The scores for the dimension D1 were the lowest when compared with all the other dimensions.

A recent study showed that the most affected dimensions were functional limitation (D1), physical pain (D2), and physical disability (D4). These findings are different from results of the present study. OHIP-14 can be applied at different stages including before, during, and after the cancer treatment. This approach allows the comparisons among various stages and may directly interfere with the results. Another study emphasized that psychological discomfort (D3) is generated by the high level of anxiety and acute pain in patients with MRONJ (19). Since the present study was a cross-sectional study, OHIP-14 was applied only after the presence of MRONJ was confirmed. This may be a limitation of the present study, since comparisons during the other stages in the same patients was not possible.

The imaging characteristics of MRONJ are more frequently observed in the more advanced stages where it 
is possible to observe changes such as a larger area of bone involvement, painful symptoms, and neurosensitive alterations, which result in alterations in chewing and swallowing and significant deterioration in the QOL. $(20,21)$

The stagewise classification facilitates the diagnosis and the therapeutic management of MRONJ (1). Among the 4 classification stages ( 0 , I, II, III), stage I was the most prevalent in the present study, followed by stage II and stage 0 . Stage III was not observed. The treatment of MRONJ aims to reduce the painful symptoms, control infection, and reestablish social contact with family and friends, thus improving the physical and mental state and consequently improving the QOL of these individuals $(11,22)$.

QOL was negatively affected by psychological discomfort in our study with a strong correlation $(p<0.001)$, which we highlight as a differential $(5,23)$. Since concern and stress is associated with patients' oral health and osteonecrosis symptoms, it is important to develop a multidisciplinary treatment approach with medical, dental, and psychological support to minimize the impact of MRONJ on the QOL.

The best way to assess the impact of oral health on the QOL would be to apply the OHIP-14 before and after the MRONJ treatment and compare the results. This approach was not possible in the present study, as some patients died due to disease complications.

The results of the present study showed that MRONJ negatively affects the oral health of the patients, especially with respect to psychological discomfort. The OHIP-14 proved to be an effective tool in assessing the impact of oral problems on the QOL.

\section{References}

1. Ruggiero SL, Dodson TB, Fantasia J, Goodday R, Aghaloo T, Mehrotra B, et al. American Association of Oral and Maxillofacial Surgeons position paper on medication-related osteonecrosis of the jaw - 2014 update. J Oral Maxillofac Surg. 2014;72:1938-56.

2. Capocci M, Romeo U, Guerra F, Mannocci A, Tenore G, Annibali $\mathrm{S}$, et al. Medication-related osteonecrosis of the jaws (MRONJ) and quality of life evaluation: a pilot study. Clin Ter. 2017;168:e253-7.

3. Barrios R, Bravo M, Gil-Montoya JA, Martínez-Lara I, García-Medina B, Tsakos G. Oral and general health-related quality of life in patients treated for oral cancer compared to control group. Health Qual Life Outcomes. 2015;13:1-8.

4. Slade GD. Derivation and validation of a short-form oral health impact profile. Community Dent Oral Epidemiol. 1997;25:284-90.

5. Miksad RA, Lai K-C, Dodson TB, Woo S-B, Treister NS, Akinyemi $\mathrm{O}$, et al. Quality of life implications of bisphosphonate-associated osteonecrosis of the jaw. Oncologist. 2011;16:121-32.

6. Stuani VT, Santos PSS, Damante CA, Zangrando MSR, Greghi SLA, Rezende MLR, et al. Oral health impact profile of head and neck cancer patients after or before oncologic treatment: an observational analytic case-control study. Support Care Cancer. 2018;26:2185-9.

7. Khan AA, Morrison A, Hanley DA, Felsenberg D, McCauley LK, O'Ryan F, et al. Diagnosis and management of osteonecrosis of the jaw: a systematic review and international consensus. J Bone Miner Res. 2015;30:3-23.

8. Nicolatou-Galitis O, Schiodt M, Mendes RA, Ripamonti C, Hope S, Drudge-Coates L, et al. Medication-related osteonecrosis of the jaw: definition and best practice for prevention, diagnosis, and treatment. Oral Surg Oral Med Oral Pathol Oral Radiol. 2019;127:117-35.

9. Kyrgidis A, Triaridis S, Kontos K, Patrikidou A, Andreadis C, Constantinidis J, et al. Quality of life in breast cancer patients with bisphosphonate-related osteonecrosis of the jaws and patients with head and neck cancer: a comparative study using the EORTC QLQ-C30 and QLQ-HN35 questionnaires. Anticancer Res. 2012;32:3527-34.

10. Hendriks LE, Hermans BC, Van Den Beuken-van Everdingen MH, Hochstenbag MM, Dingemans AM. Effect of bisphosphonates, denosumab, and radioisotopes on bone pain and quality of life in patients with non-small cell lung cancer and bone metastases: a systematic review. J Thorac Oncol. 2016;11:155-73.

11. Oteri G, De Ponte FS, Runci M, Peditto $\mathrm{M}$, Marcianò $\mathrm{A}$, Cicciù $\mathrm{M}$. Oral-health-related quality of life after surgical treatment of osteonecrosis of the jaws. J Craniofac Surg. 2018;29:403-8.

12. Ware JE, Kosinski M, Keller SD. A 12-Item Short-Form Health Survey: construction of scales and preliminary tests of reliability and validity. Med Care. 1996;34:220-33.

13. Barrios R, Tsakos G, García-Medina B, Martínez-Lara I, Bravo M. Oral health-related quality of life and malnutrition in patients treated for oral cancer. Support Care Cancer. 2014;22:2927-33.

14. Karbach J, Al-Nawas B, Moergel M, Daubländer M. Oral health-related quality of life of patients with oral lichen planus, oral leukoplakia, or oral squamous cell carcinoma. J Oral Maxillofac Surg. 2014;72:1517-22.

15. Shavi GR, Thakur B, Bhambal A, Jain S, Singh V, Shukla A. Oral health related quality of life in patients of head and neck cancer attending cancer hospital of Bhopal City, India. J Int oral Health. 2015;7:21-7.

16. Santos PSS, Cremonesi AL, Quispe RA, Rubira CM. The impact of oral health on quality of life in individuals with head and neck cancer after radiotherapy: the importance of dentistry in psychosocial issues. Acta Odontol Latinoam. 2017;30:62-7.

17. Kam D, Salib A, Gorgy G, Patel TD, Carniol ET, Eloy JA, et al. Incidence of suicide in patients with head and neck cancer. JAMA Otolaryngol Head Neck Surg. 2015;141:1075-81.

18. De Oliveira BH, Nadanovsky P. Psychometric properties of the Brazilian version of the Oral Health Impact Profile-short form. Community Dent Oral Epidemiol. 2005;33:307-14.

19. Sturrock A, Preshaw PM, Hayes C, Wilkes S. Perceptions and attitudes of patients towards medication-related osteonecrosis of the jaw (MRONJ): a qualitative study in England. BMJ Open. 2019;9:e24376. 20. Bagan JV, Cibrian RM, Lopez J, Leopoldo-Rodado M, Carbonell E, Bagán L, et al.: Sclerosis in bisphosphonate-related osteonecrosis of the jaws and its correlation with the clinical stages: study of 43 cases. Br J Oral Maxillofac Surg, 2015;53:257-62.

21. Ribeiro GH, Chrun ES, Dutra KL, Daniel FI, Grando LJ. Osteonecrosis of the jaws: a review and update in etiology and treatment. Braz J Otorhinolaryngol. 2017;pii: S1808-8694:30097-6.

22. Farias D, Zen Filho EZ, de Oliveira TF, Tinôco-Araújo JE, Sampieri $\mathrm{MB}$, et al. Clinical and image findings in bisphosphonate-related osteonecrosis of the jaws. J Craniofac Surg. 2013;24:1248-51.

23. Rathbone EJ, Brown JE, Marshall HC, Collinson M, Liversedge V, Murden GA, et al. Osteonecrosis of the jaw and oral health-related quality of life after adjuvant zoledronic acid: an adjuvant zoledronic acid to reduce recurrence trial subprotocol (BIG01/04). J Clin Oncol. 2013;31:2685-91.

\section{Acknowledgements}

The present work was carried out with the support of the National Council of Scientific and Technological Development - Brazil.

The statistical analysis of the present study was carried out with the support of PhD. José Roberto Pereira Lauris.

\section{Conflict of Interest}

No competing financial interests exist. 